\title{
Comparison of type-2 fuzzy inference method and deep neural networks for mass detection from breast ultrasonography images
}

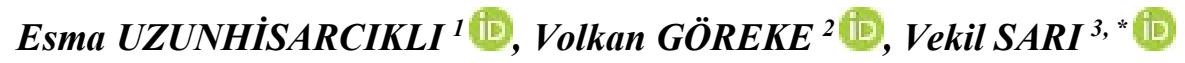 \\ ${ }^{1}$ Erciyes University, Kayseri Vocational College, Kayseri/ TURKEY \\ ${ }^{2}$ Sivas Cumhuriyet University, Sivas Vocational College, Sivas/ TURKEY \\ ${ }^{3}$ Sivas Cumhuriyet University, Faculty of Engineering, Department of Electrical and Electronics Engineering, Sivas/ TURKEY
}

\begin{abstract}
In this study, mass detection from breast ultrasonography images was realized using deep neural networks. Dataset is a collection of publicly available ultrasonography images which were classified by their biopsy results. A total of 153 breast ultrasonography images that contain 89 malign and 64 benign tumours were used. Image augmentation and deep neural network software was developed using Python 3,5 environment on Visual Studio Community 2017 IDE. A hybrid method including Keras ImageDataGenerator Class and image preprocessing techniques was introduced. Twenty images from both classes were randomly split from the dataset for testing after the network was designed. The network had a success rate of $100 \%$ at an epoch value of 70 . The result of this study was compared with the result of another study that implemented type-2 fuzzy inference system with a success rate of $99,34 \%$. As a conclusion, it can be expressed that the deep neural networks are more successful than fuzzy inference systems in tumour detection from breast ultrasonography images. Therefore, it can be more convenient to use deep neural network technology in computer aided detection systems for mass detection from breast ultrasonography images.
\end{abstract}

\section{Article info}

History:

Received: 20.02 .2020

Accepted: 29.11 .2020

Keywords:

Breast cancer,

Ultrasonography, CAD, Deep neural networks.

\section{Introduction}

Cancer is defined as the abnormal growth and reproduction of body cells. It is able to spread across different tissues and harm the surrounding ones. Breast cancer is one of the most common cancers among women and frequency of occurrence increases globally according to the statistics. Every year, more than 8 million people are diagnosed as cancer [1]. Ultrasonography (US) is a medical imaging method used by experts for characterization and detection of breast lesions and evaluation of breast cancer [2]. Additionally, because of the disadvantages of ultrasonography images such as low resolution, low contrast and spot disturbances, it is difficult to detect regions with cancer and this situation increases the dependency on the expert and causes a difference over $90 \%$ in diagnosis sensitivity among various medical institutions [3]. Computer-Aided Diagnostic (CAD) tools with features such as digital image processing and artificial intelligence both decrease the dependency to the experts and increase the diagnosis accuracy [4]. Fuzzy inference systems and deep neural networks can be used for the design of one of the fundamental components of CAD systems, namely classifier. The base of the fuzzy inference systems is constructed of the rules provided by the domain experts or extracted from numerical data [5]. Using rules, membership functions and fuzzy logic to make an inference from input data and map them to outputs is called fuzzy inference [6].

In this study, deep neural network architecture is used in a feedforward structure. This structure consists of an input layer, multiple hidden layers and an output layer. Except for the output layer, each layer in the network is connected to the next layer. This type of connection has two main features called weight average feature and activation feature [7]. Deep neural networks are a special type of networks with multiple layers and neurons. In the last decade, a type of deep neural networks called convolutional neural networks that automatically extract features have been widely used in computer vision. Deep learning techniques were also applied to the medical imaging analyses such as diagnosis of otitis media and breast lesions [8].

In this study, classification of regions with cancer using deep neural networks on breast ultrasonography 
images was implemented and the obtained results were compared with the results of a previous study that used fuzzy inference.

\section{Fuzzy Inference Systems}

Unlike classical set theory, fuzzy set theory deals with the uncertainty of the information. This theory was introduced by Zadeh in 1965. In classical set theory, the membership of an element to a set is certain ( 0 or 1) while the membership of an element in fuzzy set theory is defined with a membership function. Thus, crisp input values are transformed into non-crisp values between 0-1 using membership functions. Triangle, trapezoidal and Gaussian membership functions are the typical fuzzifiers used in this approach [9]. Fuzzy inference builds a mechanism that matches the fuzzy values of input parameters to the outputs using if-then rules. The fuzzy rules are extracted either from the dataset using statistical analysis tools or from the verbal expressions of a domain expert [10]. After type-2 fuzzy set theory was introduced by Zadeh in 1975, the first theory was entitled as type-1. Type-2 fuzzy inference system, which has a similar rule structure with type-1, has taken its actual form with Mendel's studies. Here, the structure of the membership functions differs from the previous version. The fuzzy sets of type- 1 are expressed with more than one fuzzy sets that take values between 0 and 1, and they are called with the footprint of uncertainty. The block diagram of a Type2 fuzzy inference system is given in Figure 1 [11].

This model consists of three steps: fuzzification, ifthen rules and defuzzification. In the fuzzification step, the input values are mapped to the corresponding membership values using the triangular, Gaussian and trapezoidal membership functions [12].

The general if-then rule of Mamdani algorithm is given in Equation 1 [13].

$R_{i}$ : if $x_{i}$ is $A_{i 1}$ and ... then $y$ is $B_{i}($ for $i=1,2,3, \ldots, k)$

Where $\mathrm{k}$ is the numbers of rules, $\mathrm{xi}$ is the input variable and $\mathrm{y}$ is the output variable. The first step is to calculate the degree of completion for each rule, using the membership degrees. It is given in Equation 2

$\propto_{i}=\mu_{A i 1}\left(X_{1}\right) \wedge \mu_{A i 2}\left(X_{2}\right) \wedge \ldots \wedge \mu_{A i n}\left(X_{n}\right)$

As given in Equation 3, the fuzzy output set Bi is derived for each rule using the t-norm.

$\mu_{B i}(y)=\alpha_{i} \wedge \mu_{B i}(y)$

As given in Equation 4, output fuzzy sets are aggregated using the maximum.

$\mu_{B}=\max \left(\mu_{B i}(y)\right), \quad i=1,2, \ldots, k$

As given in Equation 5, output fuzzy set is converted to the crisp value via defuzzification.

$y=\int B(y) y d y / \int B(y) d y$

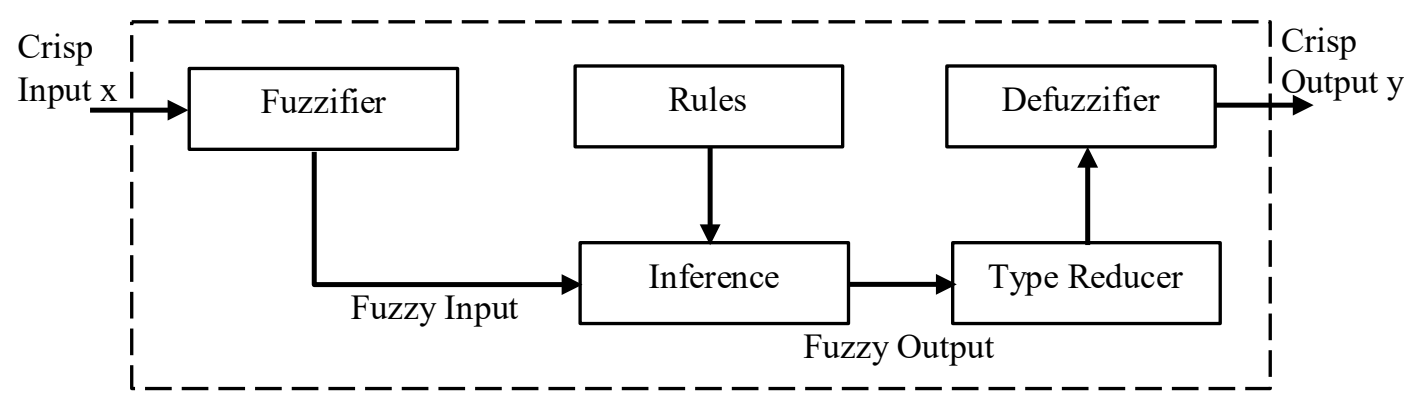

Figure 1. Type-2 fuzzy inference system. 


\section{Deep Artificial Neural Networks}

Deep learning is a special subdomain of machine learning. The first learning algorithm was introduced by Ivakhnenko and Lapa in 1965. The first study that can be considered as the basis of learning architecture was introduced by Fukushma in 1980 [14-16]. The deepness of deep learning expresses the number of hidden layers in classical neural networks. The number of layers that contribute to the data model is called the deepness of the model. Modern deep learning uses tens or even hundreds of consecutive layers. The learning process is realized via models called neural networks that were structured in real layers and stacked over themselves [17]. Deep neural networks directly extract the features for classification and detection purposes from the data. This approach is the advantage of deep

$h^{k}=\varphi\left(W^{k} h^{k-1}+b^{k}\right)$

$\hat{y}=W^{l} h^{l-1}+b^{l}$ neural networks over other machine learning methods [18]. Deep neural networks consist of three layers called input layer, hidden layer and output layer. Hidden layers implement a non-linear operation by using the output of the previous layer. The mathematical equation of the non-linear operation is given by Equation 6 . Here $\mathrm{W}^{\mathrm{k}}$ is the weight matrix of all synaptic connections among each neuron of layer $\mathrm{k}$ 1 and layer $\mathrm{k} ; \mathrm{b}^{\mathrm{k}}$ is the deviation vector in layer $\mathrm{k} ; \mathrm{h}^{\mathrm{k}-1}$ is the output of the previous layer and $\varphi(\ldots)$ is the activation function that represents the nonlinear relationship between layers. Deep neural networks normally use logistic functions $\varphi(u)=\left(1+e^{u}\right)$ inside their neurons and hyperbolic tangent functions $(\varphi(\mathrm{u})=\tanh (\mathrm{u}))$ as activation functions. Output layer is generally used for prediction and its mathematical expression is given in Equation 7 [19].

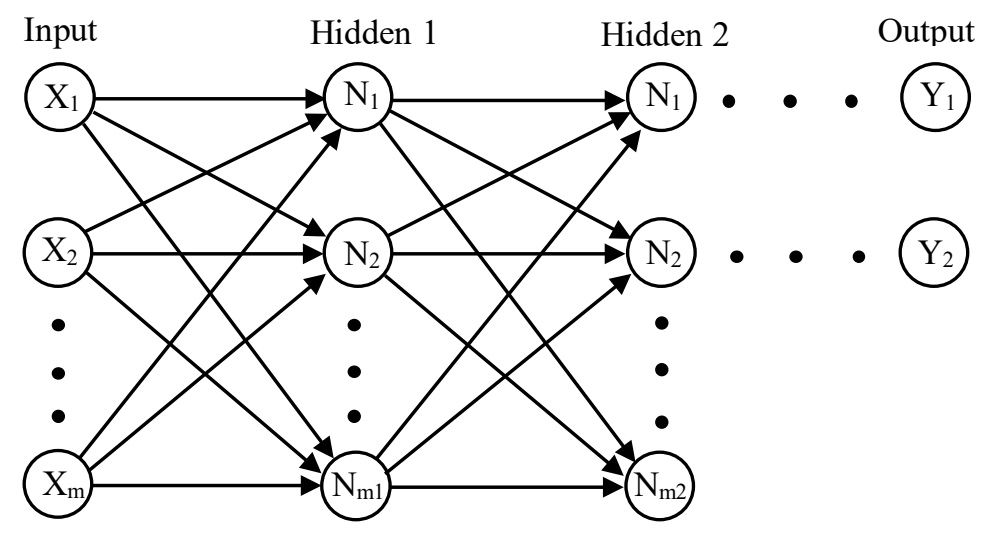

Figure 2. Deep neural network structure.

Basic components in the structure of deep neural networks are:

Weight Averaging: Amounts to average the previous layer with some weight matrix to obtain the next layer. The weight averaging procedure is as given in Equation 8 [7].

$a_{f}^{(t)(v)}=\sum_{f^{\prime}=0}^{F_{v}-1+\epsilon} \Theta_{f^{\prime}}^{(v) f} h_{f^{\prime}}^{(t)(v)}$

where $v \in[0, N-1], t \in\left[0, T_{m b}-1\right]$ and $f \in\left[0, F_{v+1}-1\right]$. The $\epsilon$ is here to include or exclude a bias term.

Activation Function: The hidden neuron of each layer is shown in Equation 9.

$h_{f}^{(t)(v+1)}=g\left(a_{f}^{(t)(v)}\right)$

where $v \in[0, N-2], f \in\left[0, F_{v+1}-1\right]$ and as usual $t \in\left[0, T_{m b}-1\right]$. Here $g$ is an activation function whose non-linearity allow to predict arbitrary output data.

The ReLU Function: The ReLU (Rectified Linear Unit) function takes its value in $[0,+\infty]$

is shown in Equation 10.

$g(x)=\operatorname{ReLU}(x)= \begin{cases}x & x \geq 0 \\ 0 & x<0\end{cases}$ 


\section{TensorFlow-Keras}

TensorFlow is a powerful open source software library for computation and it's very appropriate for large scale machine learning. Its basic principle is as follows: a simple computation graph as shown in Figure 3 is first defined in Python, then this graph is imported by TensorFlow and runs efficiently using an optimized $\mathrm{C}++$ code. TensorFlow is able to train a network with millions of parameters in a billion size dataset with millions of features. TensorFlow is developed by Google and therefore empowers most of

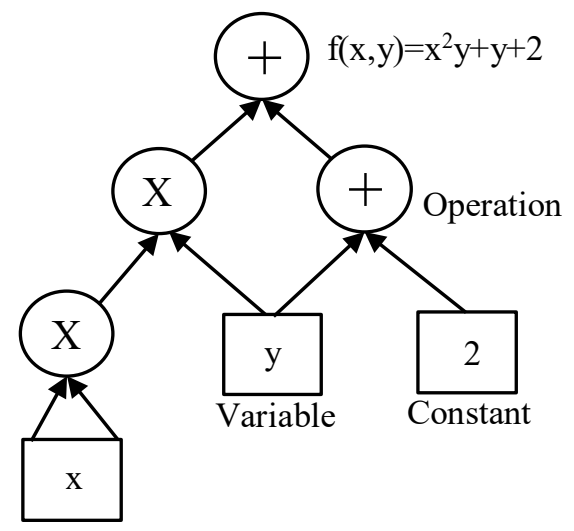

Figure 3. A simple computation graph.

\section{Materials and Methods}

In this study, a total of 153 breast ultrasonography images that contain 89 malign and 64 benign tumours were used. Image augmentation and deep artificial neural network software was developed using Python 3,5 environment on Visual Studio Community 2017 IDE. Dataset is a collection of publicly available the large-scale Google services such as Google Cloud Speech, Google Photos and Google Search. Many independent API were developed on TensorFlow such as Keras and Pretty Tensor [20]. Keras is an open source artificial neural network library developed in Python programming language. Keras requires a backend engine and can use TensorFlow, CNTK (Microsoft Cognitive Toolkit), Theano or MXNet. Using TensorFlow directly for developing is difficult because it runs in a relatively abstract level. Keras adds an easy to use abstraction level on TensorFlow [21].

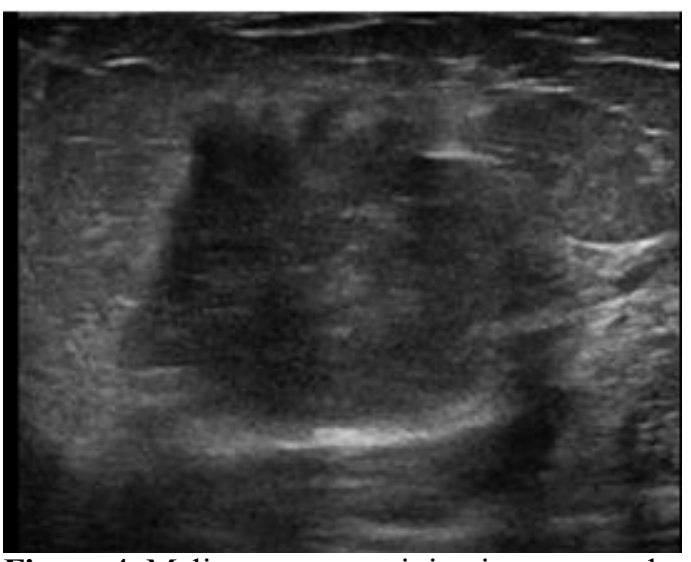

Figure 4. Malign tumour training image sample.

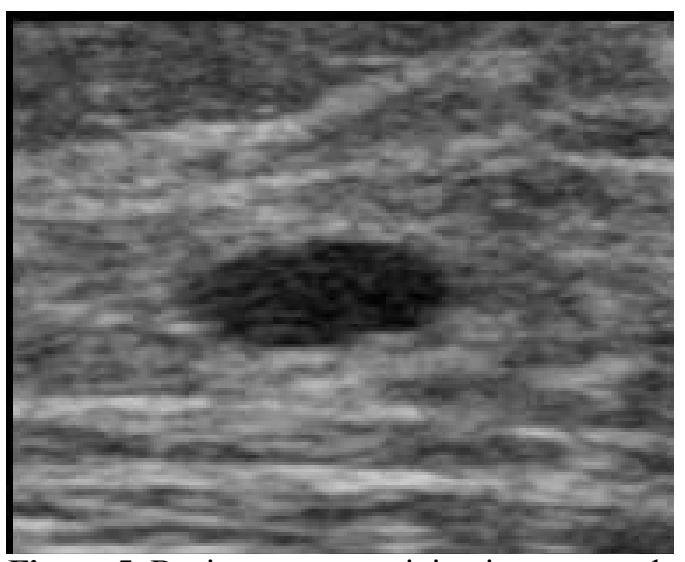

Figure 5. Benign tumour training image sample. 


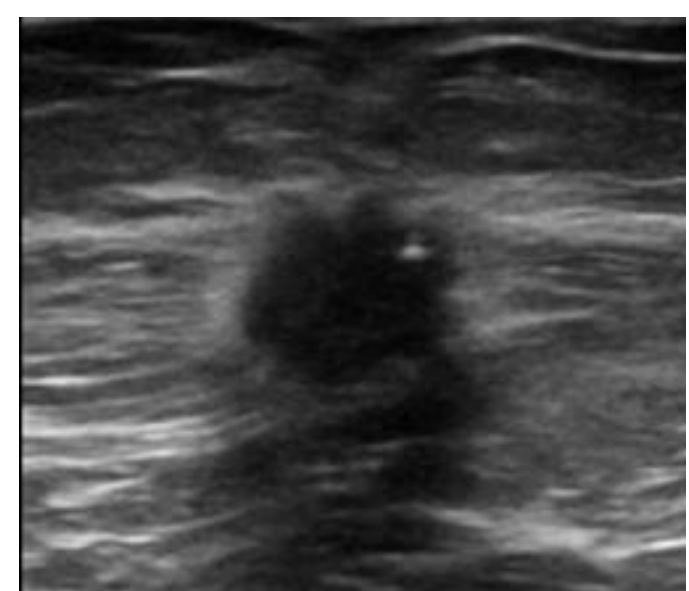

Figure 6. Malign tumour test image sample.

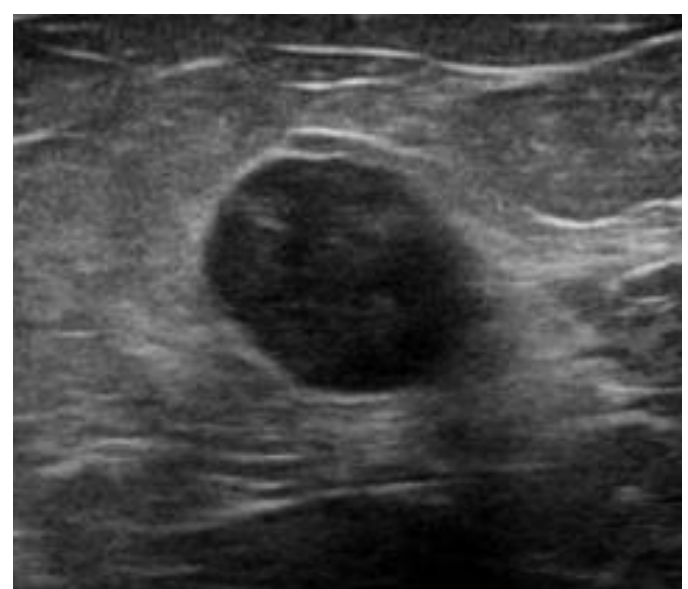

Figure 7. Benign tumour test image sample.

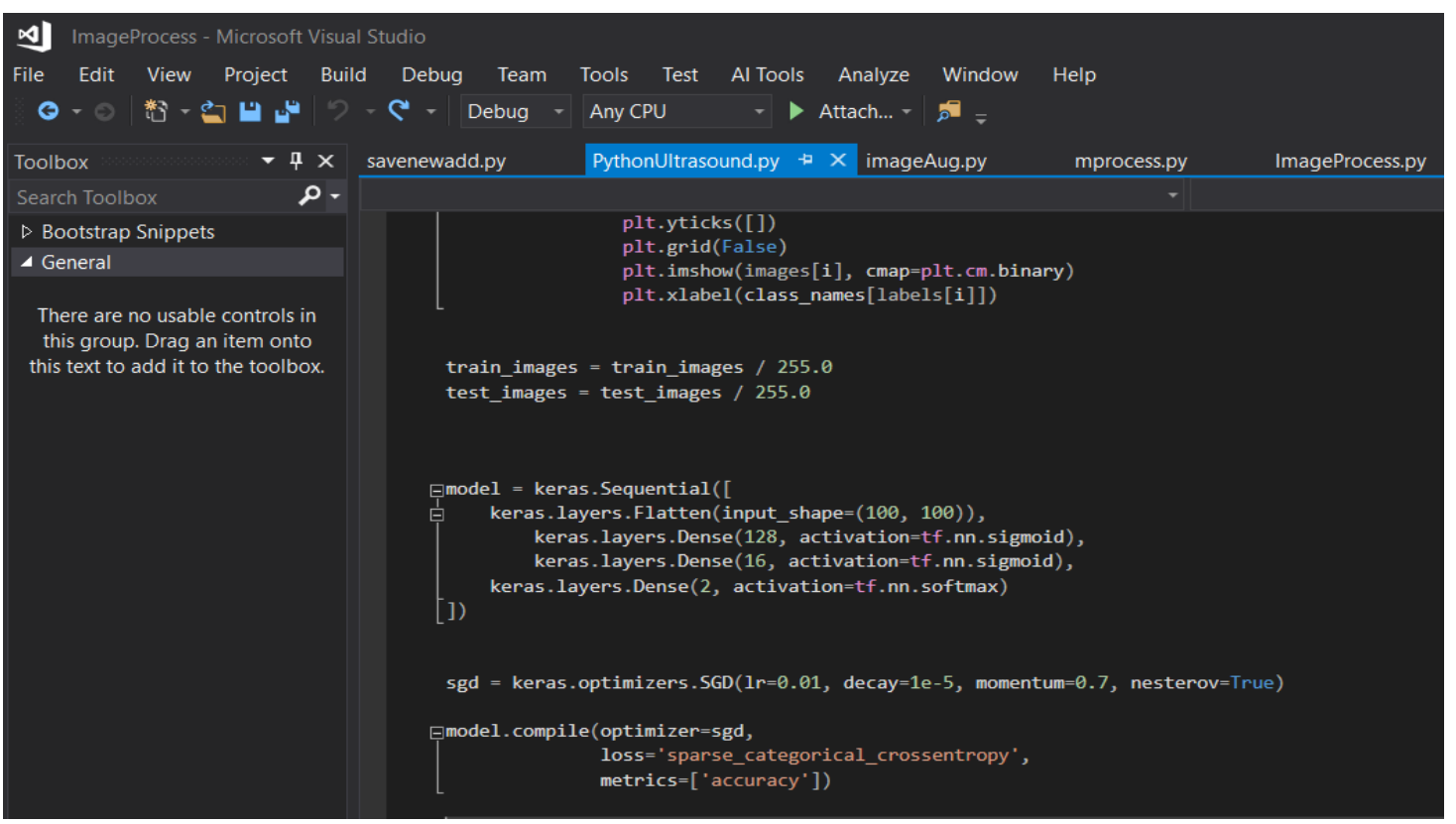

Figure 8. Software screenshot.

\subsection{Data augmentation}

Synthetic data generation is a technique that takes part in literature and is used in deep learning studies when the size of the dataset is limited. Rotation of the medical images in specific angles, shifting of the image in height and width in specific scales, inversion of the image vertically or horizontally and filtering the image are the examples of synthetic data generation. Sajjad

Table 1. Data Augmentation Methods.

\begin{tabular}{lll}
\hline No & Technique & Parameter \\
\hline 1 & Shear_range & $0.1,0.2,0.3,0.4$ \\
2 & Zoom_range & $-0.1,0.1,-0.2,0.2$ \\
3 & Brightness_range & $0.5-1.5,0.4-1.4,0.3-$ \\
& & $1.3,0.2-1.2$ \\
4 & Gauss filter(sigma) & $0.25,0.5,1,2$ \\
5 & Unsharp filter (factor) & $0.25,0.5,1.5,2$ \\
6 & Flip & Right-left \\
7 & Rotate & $-45,45,-90,90$ \\
\hline
\end{tabular}

et. al. used this technique in their study to generate 30 synthetic images from each of the 121 MR images with 8 different methods and at least 4 parameters [23, 24].

In this study, we implemented a hybrid method that consists of Keras ImageDataGenerator Class and image preprocessing techniques. Data augmentation techniques that were used are given in Table 1 together with the parameters. 


\subsection{Design and training of deep artificial neural networks}

The learning algorithm where the input data are associated with the output labels is called supervised learning [25]. In this study, the implemented deep artificial neural network uses supervised learning. The input data consist of ultrasonography images while the output labels are " 1 " and " 0 " for malign and benign tumours respectively.

The deep artificial neural network is Sequential which is one of the two models of Keras. The input layer has 128 outputs and the activation function is sigmoid. The hidden layer has 16 outputs and uses the sigmoid activation function. The last layer has 2 outputs corresponding to 2 classes and uses softmax activation function. After the network was designed, 20 images from both classes were randomly split for testing. The network was trained with an initial epoch value of 30 and it was tested iteratively.

The performance of the network was calculated using accuracy. The mathematical equation of the accuracy is given in Equation 11 [26].

Accuracy $=\mathrm{TP}+\mathrm{TN} / \mathrm{TP}+\mathrm{TN}+\mathrm{FP}+\mathrm{FN}$

Here, TP: Mass predicted as mass, FN: Mass predicted as not mass, FP: Not mass predicted as mass, TN: Not mass predicted as not mass [5]. The performance of the network is $100 \%$ at an epoch value of 70 . The screenshot of the result is shown in Figure 9.

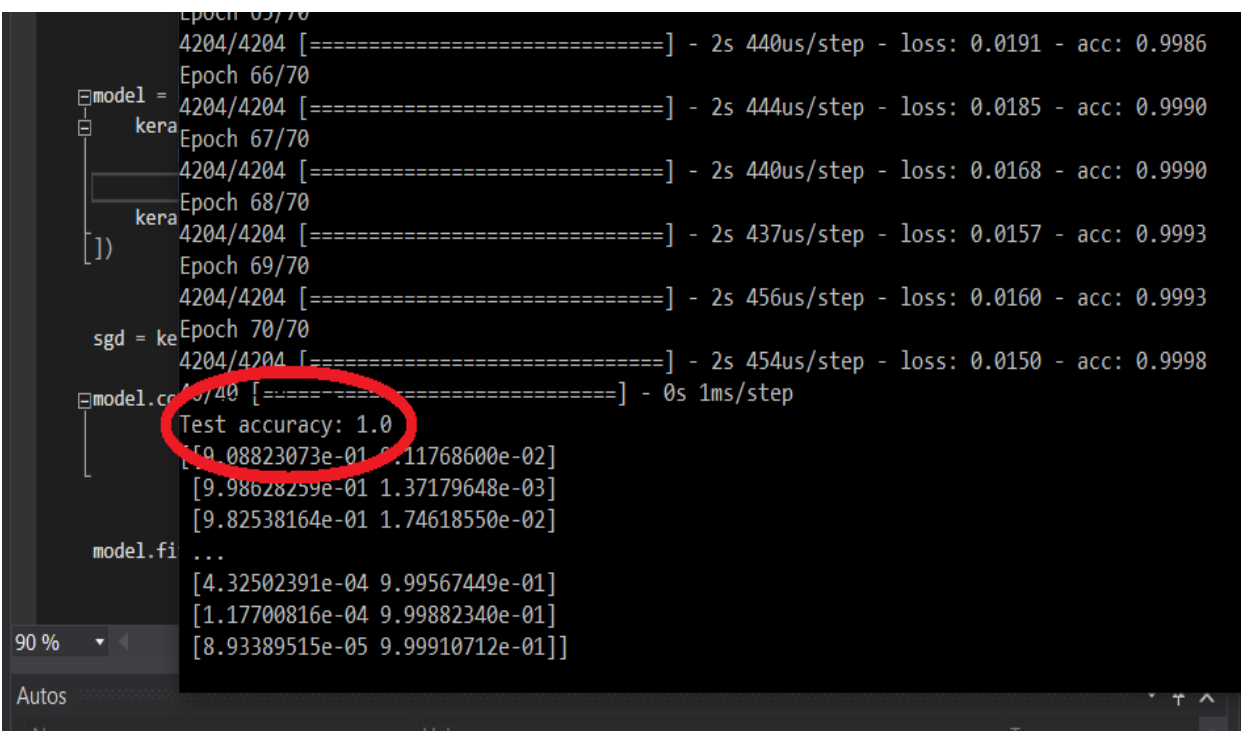

Figure 9. Screenshot of the result.

\section{Results and Discussion}

Today, artificial intelligence technologies help physicians diagnose disease. These technologies include different classification structures and are called computer-aided diagnosis (CAD) systems. These classifier structures can be based on fuzzy logic and deep neural networks. The success of CAD systems in detection can directly affect the physician's decision about the patient. Therefore, the success of the physician in diagnosis is directly related to the performance of the CAD system used. A CAD system that provides superior classification performance will positively affect the success of physicians in making the correct diagnosis about the patient.

In this study, two different classifier structures were compared to detect cancerous mass from ultrasound images of female patients. In the classifier named as Type 2 fuzzy inference system, the classifier input consists of input attribute values. In this method, feature determination and rule creation are based on the knowledge and experience of a specialist physician. In the classifier using deep neural networks, only ultrasound images form the entrance to the system. In this method, attribute determination is not required, the deep neural network structure performs this process automatically.

In a previous study, Miranda and Felipe achieved $76,76 \%$ classification success by using fuzzy logic in detecting breast cancer using ultrasound imaging. [27]. In another study conducted before, Zhang et al. achieved 93,4\% classification success using deep learning [28]. Han et al. achieved 91,23\% classification success using deep neural networks [29]. Byra et al. achieved $88.7 \%$ classification accuracy with the VGG19 deep neural network [30]. Yap et al. achieved $98 \%$ success in their deep learning study [31]. In deep neural networks study of Mohammed et al. 
$82,04 \%$ success was demonstrated [32]. Keleş et al. achieved $97 \%$ success in their study using neural fuzzy [33]. Huang et al. achieved $96,81 \%$ success in their study with the optimized fuzzy approach [34]. The success rate of Uzunhisarcikl1 and Göreke's study where they used type-2 fuzzy inference system for the detection of malign and benign tumours was $99,34 \%$ [35]. The success rate of this study with the same dataset using deep artificial neural network is $100 \%$. As a result, a superior result was obtained in this study compared to the current literature studies.

\section{Conclusion}

In this study, the classification process was carried out with deep neural networks using image data. Data set size is important in systems where deep neural networks are used. Increasing the size of the data set eliminates the problem of memorization, which is a problem for the system, and also increases the performance. In this study, filter applications designed to work with a standard data enhancement function of Keras library are applied in a hybrid way. By using the deep neural network trained with this hybrid method, a better classification performance has been obtained compared to other studies in the literature.

\section{Conflicts of interest}

The authors state that did not have conflict of interests

\section{References}

[1] Tanha J., Salarabadi H., Aznab M., Farahi A. and Zoberi M. Relationship among prognostic indices of breast cancer using classification techniques, Informatics in Medicine Unlocked, 18 (2020) 1-9.

[2] Pan H. B. The role of breast ultrasound in early cancer detection, Journal of Medical Ultrasound, 24 (4) (2016)138-141.

[3] Salomon L. J., Winer N., Bernard J. P. and Ville Y. A score-based method for quality control of fetal images at routine second-trimester ultrasound examination, Prenatal Diagnosis, 28 (9) (2008) 822-827.

[4] Yassin N. I., Omran S., El Houby E. M. and Allam $\mathrm{H}$. Machine learning techniques for breast cancer computer aided diagnosis using different image modalities: A systematic review, Computer Methods and Programs in Biomedicine, 156 (2018) 25-45.

[5] Göreke V., Uzunhisarcıklı E. and Öztoprak B. Mamogram görüntüde meme kanserine ait kitlelerin bilgisayar destekli tespiti için tip-2 bulanık çıkarım sistemi tasarımı. Tıptekno'16, Tip Teknolojileri Kongresi, 27-29 Ekim 2016, 280-284.

[6] Mamdani, E. H. Application of fuzzy algorithms for control of simple dynamic plant, Proceedings of the Institution of Electrical Engineers, 121 (12) (1974) 1585-1588.

[7] Epelbaum, T. Deep learning: Technical introduction. (2017).

[8] Chen C. H., Lee Y. W., Huang Y. S., Lan W. R., Chang R. F., Tu C. Y., Chen C. Y. and Liao W. C. Computer-aided diagnosis of endobronchial ultrasound images using convolutional neural network, Computer Methods and Programs in Biomedicine, 177 (2019) 175-182.

[9] Zadeh L. A. Fuzzy logic and approximate reasoning, Synthese, 30 (3-4) (1975) 407-428.

[10] Wang C. A study of membership functions on mamdani-type fuzzy inference system for industrial decision-making, Lehigh University, Mechanical Engineering and Mechanics, Candidacy for the Degree of Masters of Science, (2015) 199.

[11] Mendel J. M. Type-2 fuzzy sets and systems: an overview, IEEE Computational Intelligence Magazine, 2 (1) (2007) 20-29.

[12] Choi, B. I. and Rhee, F. C. H. Interval type-2 fuzzy membership function generation methods for pattern recognition, Information Sciences, 179 (13) (2009) 2102-2122.

[13] Akgun, A., Sezer, E. A., Nefeslioglu, H. A., Gokceoglu, C. and Pradhan, B. An easy-to-use MATLAB program (MamLand) for the assessment of landslide susceptibility using a Mamdani fuzzy algorithm, Computers \& Geosciences, 38 (1) (2012) 23-34.

[14] Fukushima K. Neocognitron: A self-organizing neural network model for a mechanism of pattern recognition unaffected by shift in position, Biological Cybernetics, 36 (4) (1980) 193-202.

[15] Kızrak M. A. and Bolat B. Derin öğrenme ile kalabalık analizi üzerine detaylı bir araştırma, Bilişsim Teknolojileri Dergisi, 11 (3) (2018) 263-286.

[16] Şeker A., Diri B. and Balık, H. H. Derin öğrenme yöntemleri ve uygulamaları hakkında bir inceleme, Gazi Mühendislik Bilimleri Dergisi, 3 (3) (2017) 47-64. 
[17] Chollet F. Deep Learning with Python, USA:1st ed. Manning Publications Co, 2018.

[18] Zhang Z. and Sejdić E. Radiological images and machine learning: trends, perspectives, and prospects, Computers in Biology and Medicine, 108 (2019) 354-370.

[19] Félix G., Siller M. and Alvarez E. N. A fingerprinting indoor localization algorithm based deep learning. Eighth International Conference on Ubiquitous and Future Networks, 5-8 July 2016, 1006-1011.

[20] Géron A. Hands-On Machine Learning with Scikit-Learn and TensorFlow, USA: 1st ed.: O'Reilly Media, 2017.

[21] McCaffregy J. Keras Succinctly, USA: 1st ed.: Syncfusion Inc, 2018.

[22] SonoSkills and Hitachi Medical Systems Europe. Netherlands.

Available at: https://www.ultrasoundcases.info/m ore/about-us. Retrieved February 20, 2020.

[23] Sajjad M., Khan S., Muhammad K., Wu W., Ullah A. and Baik, S. W. Multi-grade brain tumor classification using deep $\mathrm{CNN}$ with extensive data augmentation, Journal of Computational Science, 30 (2019) 174-182.

[24] Chougrad H., Zouaki H. and Alheyane O. Multilabel transfer learning for the early diagnosis of breast cancer, Neurocomputing, (2019) https://doi.org/10.1016/j.neucom.2019.01.112.

[25] Bengio Y., Goodfellow I. and Courville A. Deep learning, Book in preparation for MIT Press, 2015.

[26] Baratloo A., Hosseini M., Negida A. and El Ashal, G. Part 1: simple definition and calculation of accuracy, sensitivity and specificity, Emergency, 3 (2) (2015) 48-49.

[27] Miranda, G. H. B. and Felipe, J. C. Computeraided diagnosis system based on fuzzy logic for breast cancer categorization, Computers in biology and medicine, 64 (2015) 334-346.

[28] Zhang, Q., Xiao, Y., Dai, W., Suo, J., Wang, C., Shi, J. and Zheng, H. Deep learning based classification of breast tumors with shear-wave elastography, Ultrasonics, 72 (2016) 150-157.

[29] Han, S., Meng, Z., Khan, A. S. and Tong, Y. Incremental boosting convolutional neural network for facial action unit recognition, Advances in Neural Information Processing Systems, (2016) 109-117.

[30] Byra, M., Galperin, M., Ojeda-Fournier, H., Olson, L., O'Boyle, M., Comstock, C. and Andre, M. Breast mass classification in sonography with transfer learning using a deep convolutional neural network and color conversion, Medical physics, 46 (2) (2019) 746-755.

[31] Yap, M. H., Pons, G., Martí, J., Ganau, S., Sentís, M., Zwiggelaar, R., Davison, A. K. and Martí, R. Automated breast ultrasound lesions detection using convolutional neural networks, IEEE journal of biomedical and health informatics, 22 (4) (2017) 1218-1226.

[32] Mohammed, M. A., Al-Khateeb, B., Rashid, A. N., Ibrahim, D. A., Abd Ghani, M. K. and Mostafa, S. A. Neural network and multi-fractal dimension features for breast cancer classification from ultrasound images, Computers \& Electrical Engineering, 70 (2018) 871-882.

[33] Keleş, A., Keleş, A. and Yavuz, U. Expert system based on neuro-fuzzy rules for diagnosis breast cancer, Expert systems with applications, 38 (5) (2011) 5719-5726.

[34] Huang, Q., Hu, B. and Zhang, F. Evolutionary optimized fuzzy reasoning with mined diagnostic patterns for classification of breast tumors in ultrasound, Information Sciences, 502 (2019) 525-536.

[35] Uzunhisarcikli E. and Goreke V. A novel classifier model for mass classification using BIRADS category in ultrasound images based on Type-2 fuzzy inference system, Sädhanā, 43 (9) (2018) 138. 